\title{
O Fazer do Psicólogo do NASF e o Processo de Educação em Saúde Frente à Sexualidade na Adolescência
}

\author{
Iasminny Loiola Teixeira ${ }^{1}$; Adriana Maria Simião da Silva²
}

\begin{abstract}
Resumo: O presente estudo visa analisar através de teorizações como o psicólogo do NASF atua, no processo de educação em saúde, diante da sexualidade na adolescência. Sabe-se que o NASF é um espaço relevante para concretização de intervenções profundas no âmbito psicossocial. Além disso, é imperativo pensar na relevância do trabalho com o público adolescente, visto que esta fase da vida demanda um olhar diferenciado, a adolescência é um fenômeno relativamente novo do ponto de vista científico e empírico, sendo considerada uma fase de alterações relevantes e que repercute em vários âmbitos. Também é primal pensar no processo de Educação em Saúde como lócus central na lida com esses sujeitos tendo em vista sua pertinência como método de atuação, ou seja, mediante intervenções grupais que facilitam o processo de mediação frente ao foco desse tipo de atuação. Nesse sentido, a intervenção psicológica, partindo desses princípios, revela-se fundamental na medida em que gera mudança nos sujeitos e consequentemente espaço para atitudes mais calcadas na realidade vivida por eles e, portanto, mais conscientes.
\end{abstract}

Palavras-Chave: Psicólogo, Educação em saúde, Sexualidade.

\section{The Perform of the NASF Psychologist and the Health Education Process face to the Sexuality in Adolescents}

\begin{abstract}
The present study aims at analyzing through theories how the psychologist acts in the process of health education, in the face of sexuality in adolescence. It is known that the NASF is a relevant space for the implementation of deep interventions in the psychosocial minority. In addition, it is imperative to think about the relevance of the work with the public, given this phase of life, adolescence and a relatively new phenomenon from a scientific and empirical point of view, and a relevant development phase has repercussions in several areas. It is also essential to think about the process of health education as a central locus in dealing with these subjects in view of their relevance as a method of action, that is, through group interventions that facilitate the process of mediation in the face of this type of update. In this sense, a partial, directive psychological intervention reveals, fundamentally as it generates, changes in the subjects and consequently space for attitudes more based on the reality lived by them and, therefore, more conscious.
\end{abstract}

Keywords: Psychologist, Health education, Sexuality.

\footnotetext{
${ }^{1}$ Graduada em Psicologia no Centro Universitário Leão Sampaio (UNILEAO). Acadêmica na Especialização em Psicologia Aplicada à Educação na Universidade Regional do Cariri (URCA). E-mail: iasminnyloiola@hotmail.com.

${ }^{2}$ Doutora em Educação. Professora do Departamento de Ciências Sociais da Universidade Regional do Cariri (URCA).

E-mail: adrianamsimiao@gmail.com.
} 


\section{Introdução}

O presente estudo visa analisar através de teorizações como o psicólogo do Núcleo de Apoio à Saúde da Família (NASF) atua, no processo de educação em saúde, diante da sexualidade na adolescência. Pela apreensão histórica frente aos estudos acerca da adolescência, passando por uma análise do processo de educação em saúde no trato da temática sexualidade e adolescência, discutindo como o psicólogo atua no âmbito do NASF com foco na sexualidade adolescente buscou-se compreender como atua o psicólogo no contexto em questão.

Sabe-se que a atuação do psicólogo abrange um leque mais ou menos variado de âmbitos e de espaços. Dentre estes é possível dar destaque ao campo das intervenções psicossociais, bem como nas modalidades grupais e fundamentadas na Educação em saúde.

Paralelamente, a compreensão e o olhar frente à sexualidade do adolescente tem ganhado cada vez mais espaço, tendo em vista a crescente histórica no que tange aos estudos referentes a esta temática, bem como ao próprio lugar cedido a esta etapa da vida através de mudanças estruturais no bojo de nossa sociedade.

Assim, evidencia-se em que medida há convergência entre o saber psicológico e a intervenção com a modalidade de Educação Popular em Saúde e as questões ligadas à sexualidade de adolescentes.

Frente a tais questões é pertinente uma análise da atuação do psicólogo no contexto do NASF, apreendendo suas nuanças e perseguindo uma compreensão ampla e coesa diante de tal práxis. Além disso, é primal relacionar a vertente da Educação em saúde com a relevância das práticas grupais no manejo da escuta frente à sexualidade na adolescência.

Para tal, é relevante buscar de forma minuciosa diversos aspectos que serão apresentados na medida em que no escopo do estudo, seguindo uma linha coerente para a melhor compreensão do tema como um todo sem deixar passar, entretanto, suas especificidades. 
A relevância do trabalho do profissional psicólogo aparece como questão central ao passo que mediante a intervenção empreendida por este é que se observa a

convergência das temáticas que aqui serão trabalhadas, ou seja, o psicólogo em sua atuação tem fundamental importância na compreensão do estudo como um todo.

\section{Metodologia}

A abordagem do estudo evidencia-se como de caráter qualitativo e teórico. O foco qualitativo se dá mediante a compreensão de aspectos característicos do fenômeno em questão em detrimento de aspectos quantitativos. Além disso, por estar fundamentado eminentemente em textos clássicos e contemporâneos, evidencia-se o aspecto teórico que lhe é inerente.

Quanto aos objetivos pode-se afirmar que o presente estudo se funda como uma análise exploratória, já que visa gerar maior familiaridade com a temática em pauta, tornando-a mais explícita e possibilitando a geração de hipóteses e intuições acerca da mesma.

O presente estudo tem como procedimento a utilização da pesquisa bibliográfica, que segundo Gil (2002) consiste em utilizar material já existente como fundamento de pesquisa e suporte no que se refere à elaboração de estudos científicos. Nesse sentido, utilizam-se com maior frequência livros, artigos e textos em geral que possuam base científica.

A pesquisa bibliográfica pode ser utilizada para dar suporte a outros tipos de pesquisa que envolva aspectos empíricos, ou seja, pesquisas de campo. Entretanto, este tipo de pesquisa pode esgotar-se em si mesma.

No que diz respeito à natureza do estudo, vale citar que se configura como sendo básica, pois parte de estudos teóricos e contrapontos filosóficos para melhor apreensão da temática. Logo, não há aplicação necessariamente a partir de outros mecanismos de pesquisa.

Foi utilizado para esse estudo sites como: Scielo, Google Acadêmico e Pepsico, sendo que as palavras-chave utilizadas foram: Psicólogo no NASF, escuta psicológica, sexualidade e adolescência. Foram utilizados, também, alguns textos clássicos, tendo 
em vista a relevância dos mesmos para a construção teórica desse trabalho. Todo o estudo foi realizado durante os meses de setembro e outubro do corrente ano.

Logo, visa-se apreender a atuação do psicólogo em sua vertente crítica, como de caráter relevante e necessário diante da escuta frente à sexualidade vivenciada por adolescentes, perpassando os vários aspectos que convergem nesta temática.

\title{
A Evolução dos Estudos diante da Adolescência
}

A adolescência surge como uma categoria do desenvolvimento humano na medida em que se percebe um vácuo entre a compreensão do que seria a infância e a adultez, institucionalizando-se por estudos que revelam uma moratória diante desse estágio da vida, ou seja, evidencia-se que há uma lacuna na compreensão de como apreender todo um período do desenvolvimento humano e a partir disto surge um novo conceito (BOCK, 2007).

Existem diversas características que elucidam este momento da vida para os sujeitos, a saber, entre eles estão a busca por uma identidade própria, por uma identificação com o meio social, prevalência de tendência grupal, necessidade de intelectualizar e fantasiar, atitudes sociais reivindicatórias com características muitas vezes antissociais (ABERASTURY; KNOBEL, 1981).

É razoavelmente recente o interesse científico pelo processo de desenvolvimento que ocorre entre a infância e a adultez, ou seja, a adolescência é um processo recém descoberto como fonte de pesquisa para as ciências de modo geral (ARIÈS, 1986).

Nesse sentido para Salles (2005 p. 35),

\begin{abstract}
A identidade do adolescente é construída hoje numa cultura caracterizada pela existência de uma indústria da informação, de bens culturais, de lazer e de consumo onde a ênfase está no presente, na velocidade, no cotidiano, no aqui e no agora, e na busca do prazer imediato. A subjetividade é, então, construída no comigo mesmo, na relação com o outro, num tempo e num espaço social específicos. Mesmo que as concepções anteriores de crianças e adolescentes não possam ser descartadas porque continuam respondendo a questões presentes nessa área de estudo, é necessário problematizá-las, já que
\end{abstract}


essas concepções não permitem mais captar toda a complexidade do significado da infância e da adolescência hoje. A sociedade atual acaba por impor uma revisão da distinção entre criança, adolescente e adulto até agora dominante.

Nesse sentido é que este texto busca apontar para a necessidade de repensar os parâmetros que definem a infância e a adolescência na sociedade atual.

Após seu aparecimento enquanto momento específico do desenvolvimento humano, a adolescência sofreu transformações oriundas da cultura tornando-se uma espécie de momento de passagem. Além disso, alguns processos específicos no modo de funcionamento das famílias e da sociedade à época fundamentou miticamente a adolescência como algo natural (CALLIGARIS, 2000).

Ao que converge com o pensar de Frota (2007), visto que este ressalta que ao prescrever normas de comportamento e expressão frente a este período da vida, a sociedade aplica um pensamento mítico diante de algo que não é eminentemente natural, mas, sobretudo cultural.

Ainda no final do século XIX ocorre uma distinção no sentido de que há reconhecimento frente a esta nova categoria, a saber, a adolescência passa a ser reconhecida como um momento da vida distinto, pois não está inserida nem na infância nem na adultez (SALLES, 2005).

A partir daí surgem diversas teorizações frente à temática, inserindo-a no corpo de estudos desvelados pelas ciências, agora atrelando as particularidades desse processo da vida frente a outros, bem como pensando de forma geracional nas implicações ocorridas para aparição de novas formas de pensar o humano (MEAD, 1970).

Uma das inovações frente aos novos estudos que apareceram foi pensar o fenômeno adolescente como uma fase de "perturbações" normais diante do desenvolvimento humano, ou seja, apesar de ser uma fase de crise para os sujeitos pode ser tida como algo comum aos mesmos (KNOBEL, 1992).

Por tal prisma, evidencia-se que a caracterização do que venha a ser a adolescência surge como uma demanda da cultura para estabelecer certos padrões e lidar com as dificuldades que aparecem na realidade objetiva, ou seja, consiste em uma invenção cultural multiforme dependendo de cada cultura para ganhar significado, visto que este é único para cada uma e perpassam direitos e deveres diversos (SCHOENFERREIRA \& COLS, 2010). 


\section{A Educação em Saúde na Perspectiva de Trabalho com o Adolescente}

O processo de educação em saúde tem fundamentos relevantes no trato de várias questões ligadas à conscientização de temas diversos e ao bem estar dos sujeitos. Tratando-se da adolescência, esta prática é relevante na medida em que proporciona maior autoconhecimento e mais confiança ao lidar com as variadas situações que interpelam seu cotidiano, sendo que entre elas estão temas como sexualidade, uso e abuso de drogas, criminalidade, dentre outros (VIERO et. al., 2015).

A sexualidade é um tema constante no bojo de assuntos da adolescência, sendo aspecto central no cotidiano dos sujeitos nesta fase da vida, ou seja, é uma época em que aspectos ligados à temática sexualidade são constantes frente ao seu cotidiano e permeiam mais fortemente as relações que ele constrói nesta fase da vida (SOARES, 2008).

O abuso de drogas é um fenômeno comum à adolescência. Em nível mundial sabe-se, inclusive, que a maconha é a droga mais utilizada por sujeitos nessa fase do desenvolvimento humano. Interessante observar também que a utilização de substâncias psicoativas comumente está atrelada a outro fenômeno vivenciado na família e no meio social, a saber, a violência (MOURA; MONTEIRO; FREITAS, 2016).

O envolvimento de sujeitos na adolescência com a criminalidade é outro fato rotineiramente observado, tendo em vista que são constantemente noticiadas na grande mídia. É, por assim dizer, lugar comum o envolvimento de adolescentes em práticas ligadas à noção de criminalidade, sendo vista como uma questão social que deve ter foco nas discussões diante do crescimento social de nossa sociedade (MOREIRA; GUERRA; DRAWIN, 2017).

É relevante analisar a especificidade da atuação frente ao público em questão. Lembrando que a eficiência da intervenção está calcada em várias questões, dentre estas o foco dos próprios participantes, este depende do modo com as intervenções são dirigidas e trabalhadas, além do suporte teórico bem fundamentado do profissional e seu manejo diante do grupo (OLIVEIRA, 2009). 
A Educação em Saúde consiste em uma ferramenta primordial no que tange aos processos supracitados, visto que esta desempenha função de orientação e reflexão frente aos processos de bem-estar e de adoecimento inerentes ao estilo de vida que escolhemos trilhar, fomentando emancipação diante das próprias escolhas e melhoria na vida mediante melhor apreensão de certas atitudes (JARDIM, 2012).

A prática de atenção a adolescentes embasada na metodologia de Educação em Saúde ocupa grande espaço mediante os artigos publicados na última década. Revelando, assim, sua relevância diante da atuação, no que diz respeito ao público em questão e mesmo ao público em geral (MACEDO; CONCEICAO, 2013).

Pensando também na sexualidade adolescente, observa-se que mediante a abertura à fala ocorre conscientização frente a esta temática, ou seja, a partir do momento em que há espaço para que o adolescente expresse sua sexualidade pode ocorrer melhor apreensão da própria sexualidade (SOARES, 2008).

Assim, tem-se que uma vertente relevante para a discussão dos mais variados temas consiste na Educação em Saúde, que pode fundamentar um processo ético de emancipação para os sujeitos em suas vivências e nos modos como estes se relacionam entre si, radicalizando assim o modo de viver e compreender o mundo destes sujeitos (VIERO et. al., 2015).

Outro fenômeno que é comum à adolescência é o uso e abuso de drogas. Visto que este momento da vida é tido como uma fase de experimentação para os sujeitos é comum que ocorra o acesso a certas drogas, e que em alguns casos possa ocorrer consumo abusivo. É importante olhar para este processo tendo em vista que é rotineiro e pode gerar prejuízos irreparáveis par ao sujeito e para a sociedade como um todo (MOURA; MONTEIRO; FREITAS, 2016).

Além do que já fora discutido sobre esse período da vida, é relevante pensar também na relação do sujeito que se encontra na adolescência com práticas ligadas à criminalidade. Tendo em vista que é lugar comum a relação dúbia do adolescente com a lei, atualmente é visto com maior naturalidade e mais discernimento quando se fala em práticas infratoras cometida por adolescentes (MOREIRA; GUERRA; DRAWIN, 2017).

Dito isto, é relevante pensar na atuação profissional como mecanismo de promoção da emancipação diante de certas questões que aparecem como querelas 
sociais e que interferem diretamente no cotidiano desses sujeitos e que interpelam suas vivências em tal cotidiano (OLIVEIRA, 2009).

Nesta perspectiva, pode-se observar a modalidade de Educação em Saúde como uma vertente deveras relevante no que tange a uma melhor apreensão diante dos vários fenômenos que se evidenciam durante o período da adolescência, dentre outras questões (JARDIM, 2012).

Frente a isto o que se observa é uma crescente no que se refere ao trabalho com o público adolescente eminentemente pelo prisma da educação em Saúde, a qual visa possibilitar maior diálogo e mais conscientização diante de temáticas que aparecem como fundamentais ao adolescente na contemporaneidade (MACEDO; CONCEICAO, 2013).

\section{O Psicólogo do Nasf no Processo de Educação em Saúde Frente à Sexualidade na}

\section{Adolescência.}

A mudança sociopolítica inerente ao modelo governamental que surge nos anos dois mil no Brasil fomenta novas discussões acerca das políticas públicas de modo geral e das políticas públicas de saúde particularmente, fomentando assim a entrada do psicólogo nas diversas instituições de saúde, o que agrega perspectivas diversas na psicologia, levando-se em consideração seu modelo de formação e produção de conhecimentos, logo trata-se de uma reconfiguração da atuação psicológica (FERREIRA NETO, 2011).

Diante das diversas mudanças oriundas do período supracitado se dá a inserção do 'olhar psi' na atuação em saúde, ou seja, o profissional psicólogo começa a se inserir nos vários âmbitos relacionados ao campo da saúde como em hospitais gerais, ambulatórios, unidades básicas de saúde, Centros de Atenção Psicossocial (CAPS) e no próprio Núcleo de Apoio à Saúde da Família (NASF), dentre outros espaços de atuação (LEITE; ANDRADE; BOSI, 2013).

A atuação dos psicólogos no NASF se pauta na complementaridade em relação aos outros mecanismos de assistência em saúde, especialmente no auxílio das 
Estratégias de Saúde da Família que existem em determinado território, sendo assim o papel do psicólogo é dar suporte psicossocial diante das demandas que aparecem, mas sobretudo partir de um olhar preventivo frente ao cuidado da população assistida (OLIVEIRA et al., 2017).

Nesta perspectiva, cabe ressaltar um marco relevante, a saber, o NASF foi fundamentado ideologicamente pela portaria 154/2008 tendo como foco, o apoio à ampliação e ao aperfeiçoamento frente à atenção básica e/ou primária das famílias assistidas em determinada comunidade, perpassando assim pelas diretrizes inerentes ao Sistema de Saúde vigente em nosso país (MINISTÉRIO DA SAÚDE, 2017).

Percebe-se então, que o profissional psicólogo aparece como profissional de referência diante das atuações no âmbito do NASF, tendo em vista especialmente os cuidados diante da saúde mental dos sujeitos assistidos. Por tal prisma, evidencia-se que a intervenção psicológica é primordial no que tange ao trabalho realizado no âmbito em questão (FURTADO; CARVALHO, 2015).

Diante das questões pensadas frente ao NASF, uma das questões que se mostram evidentes é a necessidade de articulação da equipe que faz parte desta instituição para que haja bom funcionamento do sistema de atenção à saúde. Logo, o sistema de atenção e cuidado é pautado eminentemente na consonância da equipe em torno dos objetivos necessários ao bom funcionamento da atenção à saúde (CELA; OLIVEIRA, 2015).

A proposta evidenciada em tais dispositivos facilita diversos mecanismos de intervenção diante dos diversos públicos que se mostram na comunidade em questão. Dentre as intervenções pode-se pensar na Educação em Saúde como prisma de intervenção, no que tange ao público o foco é na adolescência e a temática norte diz respeito à sexualidade, ou melhor dizendo, a vivência desta.

É relevante, pois, uma explanação acerca da adolescência e de suas peculiaridades. O que se evidencia nesse sentido é que havia uma lacuna diante da compreensão do processo de desenvolvimento humano na medida em que não se percebia o processo intermediário entre a infância e a adultez (BOCK, 2007).

Após estar evidenciado a existência da adolescência observou-se que haviam certos aspectos inerentes a esse momento da vida, a saber, questões como prevalência de tendência grupal, necessidade de intelectualizar e fantasiar, atitudes sociais 
reivindicatórias com característica muitas vezes antissociais, busca por uma identidade própria, por uma identificação com o meio social (ABERASTURY; KNOBEL, 1981).

Um ponto relevante a ser discutido é que de algum modo as culturas apreendem a adolescência de modo distinto dando significados e sentidos diversos a este momento, ou seja, o processo de vivenciar a adolescência depende do aporte cultural no qual o sujeito está inserido (CALLIGARIS, 2000).

A sexualidade é talvez o ponto mais relevante frente ao momento da adolescência, ao menos do ponto de vista das discussões que estes têm, pois sempre é tema de debate para os jovens que começam a perceber seu corpo, que começam a pensar em suas funcionalidades e nas sensações que muitas vezes podem ser confusas (SOARES, 2008).

Evidencia-se que para os profissionais que desejam trabalhar com adolescentes na atenção básica, estes precisam saber manejar a intervenção quando o tema em questão é sexualidade, tendo em mente que é assunto recorrente frente aos grupos de adolescentes (CANO, FERRIANI, 2000).

Existe interesse por ambos os gêneros quando se trata de discutir a sexualidade, dos dois lados há curiosidade e vontade de entender melhor acerca de tal tema e de suas implicações, mas é válido citar que há diferenças, por exemplo, nos significados e sentidos atrelados para cada gênero, como também o nível de conhecimento aceitável sobre o tema é diferente entre meninos e meninas (BRETÂS et. al., 2011).

$\mathrm{Na}$ realidade esta temática deveria ter centralidade nas intervenções psicossociais frente aos jovens e à família, pois principalmente no meio familiar não há orientações claras diante da sexualidade. Os adolescentes, por sua vez, perpassam informações dissonantes da realidade por não terem sido corretamente ensinados pela célula social primária. Este processo gera problemas dos mais diversos para esses sujeitos e mesmo para a sociedade. Daí a relevância de intervenções pelas políticas de atenção básica em saúde (COSTA, 2001). 


\section{Considerações Finais}

Frente à discussão empreendida no presente estudo é possível levantar algumas questões, não como fechamento, mas como abertura para novas possibilidades, como interpelações que suscitem novas curiosidades e consequentemente novos estudos diante da atuação do psicólogo no NASF frente aos meandros da adolescência e de sua sexualidade.

É fato que há diversos estudos que tratam da temática aqui posta em foco, entretanto algumas ainda são metodologicamente confusas, evidenciando ser necessário crescimento diante desta questão. Além disso, é possível observar que há certo malestar pairando sobre o tema sexualidade, especialmente no âmbito familiar.

Um fato interessante analisado em muitos estudos, consiste nas dificuldades enfrentadas pelos profissionais de psicologia diante de querelas advindas da própria estruturação frente às demandas de sua atuação, carga horária exacerbada e dissonância entre a teoria e as prática em equipes multiprofissionais.

A adolescência sofreu mudanças de paradigma, de sua compreensão em nossa sociedade e nos modos como é vivenciada e compreendida pelos sujeitos em geral, portanto, é primordial um olhar atualizado diante do modo como este fenômeno se mostra, assim, evidencia-se a necessidade de uma formação continuada para o profissional de psicologia.

Além disso, a sexualidade é um tema recorrente em todas as classes sociais, mediante a todos os gêneros e idades, dito isto é necessária a atenção para que o profissional permita em sua intervenção o diálogo aberto, livre de estereótipos e/ou preconcepções perante tal tema e perante os sujeitos que estão sendo assistidos.

Observa-se até aqui como é imprescindível tratar com seriedade as questões da sexualidade na adolescência, sobretudo quando se pensa nas camadas mais pobres da população, que têm menor acesso aos meios de educação frente a diversos temas, mas sobretudo no que tange às questões envoltas no bojo da sexualidade humana.

Logo, o papel do NASF e de seus profissionais é observado como primordial, radicalmente necessário tendo em vista que as ações primárias em saúde têm grande chance de mudar aspectos negativos em relação aos grupos e aos sujeitos assistidos. $\mathrm{O}$ papel do psicólogo, então, aparece como pilar para que haja êxito diante das propostas inerentes a este mecanismo de bem estar psicossocial. 
Auxiliar na compreensão da sexualidade pelos adolescentes de uma dada área é trabalhar o afeto no âmago de sua plenitude, é fortalecer vínculos pelo diálogo comum, é conscientizar para transformar e, por sua vez, é sobretudo transformar a sociedade para algo novo e diferente, para uma perspectiva que abra os olhos para si e para a alteridade.

Não se pretendeu, com o presente estudo, esgotar os questionamentos frente à temática aqui analisada, mas fomentar as discussões no que se refere à mesma, frente à sua relevância e à necessidade de novos paradigmas para o enfrentamento de um problema tão antigo e tão negligenciado.

\section{Referências}

ABERASTURY, A.; KNOBEL, M. Adolescência normal: Um enfoque psicanalítico. Porto Alegre: Artes Médicas, 1981.

ARIÈS, P. História social da infância e da família. Rio de Janeiro: Guanabara, 1986.

BOCK, Ana Mercês Bahia. A adolescência como construção social: estudo sobre livros destinados a pais e educadores. Psicol. Esc. Educ. (Impr.), Campinas , v. 11, n. 1, p. 63-76, June 2007 . Available from <http://www.scielo.br/scielo.php?script=sci arttext\&pid =S1413$85572007000100007 \& \operatorname{lng}=$ en\&nrm=iso $>$. access on 30 de setembro de 2017.

BRETAS, José Roberto da Silva et al. Aspectos da sexualidade na adolescência. Ciênc. saúde coletiva [online]. 2011, vol.16, n.7 [cited 2018-01-03], pp.3221-3228. Available from: $<$ http://www.scielo.br/scielo.php?script=sci_arttext\&pid=S1413812320110008 $00021 \& \operatorname{lng}=$ en\&nrm=iso>.

CALLIGARIS, C. A adolescência. São Paulo: Publifolha, 2000.

CANO, M.A.T.; FERRIANI, M.das G.C. Sexualidade na adolescência: um estudo bibliográfico. Rev.latinoam.enfermagem, Ribeirão Preto, v. 8, n. 2, p. 18-24, abril 2000. Disponível em: < http://www.scielo.br/pdf/rlae/v8n2/12413 >. Acesso em 03 de janeiro de 2017.

CELA, Mariana. OIVEIRA, Isabel Fernandes de. O psicólogo no Núcleo de Apoio à saúde da Família: articulação de saberes e ações. Estudos de Psicologia, 20(1), janeiro a março de 2015, 31-39. Disponível em: < http://www.scielo.br/pdf/epsic/v20n1/1413-294X -epsic-20-010031.pdf >.

COSTA, M. C. O. Sexualidade na adolescência: desenvolvimento, vivência e propostas de intervenção. Jornal de Pediatria - Vol. 77, Supl.2, 2001. Disponível em: < http://scholar.google.com.br/scholar_url?url=http://xa.yimg.com/kq/groups/24183809/10885037 

AM0QgAM IKSgCMAA >. Acessos em 03 de janeiro de 2017.

FERREIRA NETO, J.L. Psicologia, políticas públicas e o SUS. São Paulo: Escuta, 2011.

FERREIRA, Márcia de Assunção. A educação em saúde na adolescência: grupos de discussão como estratégia de pesquisa e cuidado-educação. Texto contexto - enferm., Florianópolis , v. 15 , n. 2, p. 205-211, June 2006 . Available from $<$ http://www.scielo.br/ scielo.php?script=sci arttext\&pid=S0104- 07072006000200003\&lng=en\&nrm=iso>. access on 12 Sep. 2017.

FROTA, Ana Maria Monte Coelho. Diferentes concepções da infância e adolescência: a importância da historicidade para sua construção. Estud. pesqui. psicol., Rio de Janeiro , v. 7, n. 1, jun. 2007 . Disponível em <http://pepsic.bvsalud.org/scielo.php?script=sci_ arttext\&pid=S1808- 42812007000100013\&lng=pt\&nrm=iso>. acessos em 30 set. 2017.

JARDIM, Dulcilene Pereira. Educação em saúde na adolescência: uma experiência acadêmica na Estratégia Saúde da Família. Revista Oficial do Núcleo de Estudos da Saúde do Adolescente / UERJ - Vol. $9 \mathrm{n}^{\mathrm{o}} 4$ - Out/Dez - 2012. Disponível em: < http://www.adolescenciaesaude.com/detalhe_artigo.asp?id=347>. Acessos em 24 de setembro de 2017.

KNOBEL, M. A síndrome da adolescência normal. In: ABERASTURY, A.; KNOBEL, M. Adolescência normal. 10. ed. Porto Alegre, RS: Artes Médicas, 1992. p. 2462.

LEITE, Débora Cabral; ANDRADE, Andréa Batista; BOSI, Maria Lúcia Magalhães. A inserção da Psicologia nos Núcleos de Apoio à Saúde da Família. Physis, Rio de Janeiro, v. 23, n. 4, p. 1167- 1187, Dec. 2013 Available from $<$ http://www.scielo.br/scielo.php?script=sci arttext\&pid=S0103$73312013000400008 \& \operatorname{lng}=$ en\&nrm=iso> . access on 03 Jan. 2018.

MACEDO, Etiene Oliveira Silva de; CONCEICAO, Maria Inês Gandolfo. Ações em grupo voltadas à promoção da saúde de adolescentes. Rev. bras. crescimento desenvolv. hum., São Paulo , v. 23, n. 2, p. 222-230, 2013 . Disponível em<http://pepsic. bvsalud.org/scielo.php?script=sci_arttext\&pid=S0104-

12822013000200016\&lng=pt\&nrm=iso>. acessos em 25 Set. 2017.

MEAD, M. O conflito de gerações. Lisboa: Dom Quixote, 1970.

MINISTÉRIO DA SAÚDE. (2017). Portaria GM no 154, de 24 de janeiro de 2008. Cria os Núcleos de Apoio à Saúde da Família-NASF. Brasília, DF: Autor. Disponível em: < http://189.28.128.100/dab/docs/legislacao/portaria154_24_01_08.pdf >. Acesso em 03 de janeiro de 2017.

MOURA, Natana Abreu; MONTEIRO, Ana Ruth Macêdo; FREITAS, Rodrigo Jácob Moreira. Adolescents using (il)licit drugs and acts of violence. Journal of Nursing UFPE on line ISSN: 1981- 8963, [S.1.], v. 10, n. 5, p. 1685-1693, apr. 2016. ISSN 1981-8963. Available at: <https://periodicos.ufpe.br/revistas/revistaenfermagem /article/view/13543>. Date accessed: 05 dec. 2017. 
OLIVEIRA, Carla Braga et al . As ações de educação em saúde para crianças e adolescentes nas unidades básicas da região de Maruípe no município de Vitória.Ciênc. saúde coletiva, Rio de Janeiro , v. 14, n. 2, p. 635-644, Apr. 2009 . Available from

$<$ http://www.scielo.br/scielo.php?script=sci_arttext\&pid=S1413-

81232009000200032

\&lng=en\&nrm=iso $>$. access on 12 Sep. 2017.

OLIVEIRA, Isabel Fernandes de et al . The role of the psychologist in NASF: challenges and perspectives in primary health care. Temas psicol., Ribeirão Preto , v. 25, n. 1, p. 291-304, mar. $2017 \quad$ Disponível em $<$ http://pepsic.bvsalud.org/scielo.php?script=sci_arttext\&pid=S1413-

89X2017000100017\&lng=pt\&nrm=iso>. acessos em 03 jan. 2018.

SALLES, Leila Maria Ferreira. Infância e adolescência na sociedade contemporânea: alguns apontamentos. Estud. psicol. (Campinas)[online]. 2005, vol.22, n.1, pp.33-41. ISSN 19820275. Disponível em: < http://www.scielo.br/scielo.php?pid=S0103166X2005000100005\&script=sci_abstract\&tlng=pt >. Acesso em 20 de setembro de 2017.

SOARES, Sônia Maria et al . Oficinas sobre sexualidade na adolescência: revelando vozes, desvelando olhares de estudantes do ensino médio. Esc. Anna Nery, Rio de Janeiro, v. 12, n. 3, p. 485-491, Sept. 2008 Available from <http://www.scielo.br/scielo.php?script=sci_arttext\&pid=S1414-

81452008000300014\&lng=en\&nrm=iso>. access on 05 Dec. 2017.

T. H. Schoen-Ferreira \& Cols. Adolescência através dos Séculos. Psicologia: Teoria e Pesquisa Abr-Jun 2010, Vol. 26 n. 2, pp. 227-234. Disponível em: < http://www.repositorio.unifesp.br/bitstream/handle/11600/5810/S010237722010000200004.pdf?sequence=1 >. Acesso em 7 de outubro de 2017.

VIERO, Vanise dos Santos Ferreira; FARIAS, Joni Marcio de; FERRAZ, Fabiane; SIMÕES, Priscyla Waleska; MARTINS, Jéssica Abatti; CERETTA, Luciane Bisognin. Educação em saúde com adolescentes: análise da aquisição de conhecimentos sobre temas de saúde. Esc Anna Nery 2015;19(3):484-490 - Universidade do Extremo Sul Catarinense. Criciúma - SC, Brasil. Disponível em: < http://www.scielo.br/pdf/ean/ v19n3/1414-8145-ean-19-03-0484.pdf >. Acesso em 5 de outubro de 2017.

\section{Como citar este artigo (Formato ABNT):}

TEIXEIRA, Iasminny L.; SILVA, Adriana S. da. O Fazer do Psicólogo do NASF e o Processo de Educação em Saúde Frente à Sexualidade na Adolescência. Id on Line Revista Multidisciplinar e de Psicologia, 2018, vol.12, n.39, p. 772-785. ISSN: 1981-1179.

Recebido: 26.01.2018

Aceito: 29.01.2018 